\title{
IN DEFENCE OF THE INTRINSIC DEFLECTION
}

\author{
BY \\ GORDON E. DOWER \\ From the Department of Pharmacology, University of British Columbia, Canada*
}

Received June 12, 1961

The existence of a component of the epicardial electrocardiogram that can be properly called the intrinsic deflection, to use Lewis's term (Lewis and Rothschild, 1915), is a matter of both importance and dispute. Whether or not intrinsicoid deflections (MacLeod et al., 1930) occur in præcordial leads is of considerable interest to vectorcardiographers, most of whom base their work on the belief that events represented in the electrocardiogram find themselves, to a greater or lesser extent, represented in the corresponding vectorcardiogram. The significance of correlation between the ECG and the VCG has been pointed out by Duchosal and Sulzer (1949). The introduction of more advanced vectorcardiographic lead systems has improved such correlation (Sano et al., 1959) and certain cancellation experiments have indicated that on theoretical grounds, with accurate image space data for the individual examined, almost perfect correlation might be achieved (Frank, 1959). This view has not, however, been universally accepted; for example, McFee and Parungao (1959) have cast some doubt upon previous interpretations of cancellation experiments. Whether all significant electrocardiographic data provided by the standard 12-lead electrocardiogram were present in the X, Y, Z-orthocardiograms obtained from the SVEC III lead system (Schmitt and Simonson, 1955), was recently investigated in an interesting manner by Pipberger et al. (1961) who used a lead resolver to reconstruct the 12-lead electrocardiogram from the $3 \mathrm{X}, \mathrm{Y}, \mathrm{Z}$-orthocardiograms and presented the standard 12-lead and the reconstructed 12-lead electrocardiograms for clinical interpretation. Differences were insignificant.

This would suggest that the existence of an intrinsicoid deflection in præcordial leads is unlikely. That the concept of the intrinsic deflection itself was also in error was given direct experimental support by Sano et al. (1956), who compared the intrinsic deflection in direct leads taken from the frog's heart with the upstroke of the transmembrane potential curve simultaneously obtained from an intracellular microelectrode, recording from the same point of the heart surface. These workers took the point of inflection of the upstroke of the monophasic curve obtained from an intracellular electrode to indicate activation of the area beneath a surrounding ring electrode that recorded the electrocardiogram containing the "intrinsic deflection". A complete lack of apparent correlation was found and this was taken to indicate that the concept of intrinsic deflection as originally proposed by Lewis was spurious. Because it would seem unarguable that the transmembrane potential curve obtained from an intracellular microelectrode does in fact indicate activation of the cell penetrated, the method employed by Sano et al. (1956) to prove the intrinsic deflection appears unassailable. Furthermore, so important was their finding that it appeared worthy of confirmation on mammalian heart.

Details of the experimental work to be described appear elsewhere (Dower and Osborne, 1958; Dower and Geddes, 1960). The guinea-pig heart was studied in vivo. In the first phase of the work the usefulness of an intracellular recording technique to determine surface activation of the ventricles

\footnotetext{
* This work was aided by grants from the National Research Council of Canada and the British Columbia Heart Foundation.
} 
was established. All external surfaces were explored with the floating microelectrode technique of Woodbury and Brady (1956) and fast paper speeds $(75-150 \mathrm{~cm} . / \mathrm{sec}$.) were employed so that the upstroke of the monophasic curve could be accurately timed. This upstroke, according to ionic theory, results from the inflow of sodium ions into the cell and marks its activation (Cranefield and Hoffman, 1958). In the in vivo preparation, however, when the intracellular electrode voltage changes are compared with a remote reference such as the left leg, it is found that the upstrokes of the monophasic curves, instead of being evenly sigmoidal such as would be expected from ionic theory and is generally found in in vitro preparations, are curiously irregular (Dower and Osborne, 1958). The irregularities were of three types and are shown in Fig. 1. It can be seen that the upstroke possesses a steep part and gradual parts which may precede or follow the steep upstroke or both. The contribution to the total height of the action potential derived from the steep part of the upstroke is usually about 50 per cent. The duration of the total rise is approximately 16 milliseconds-
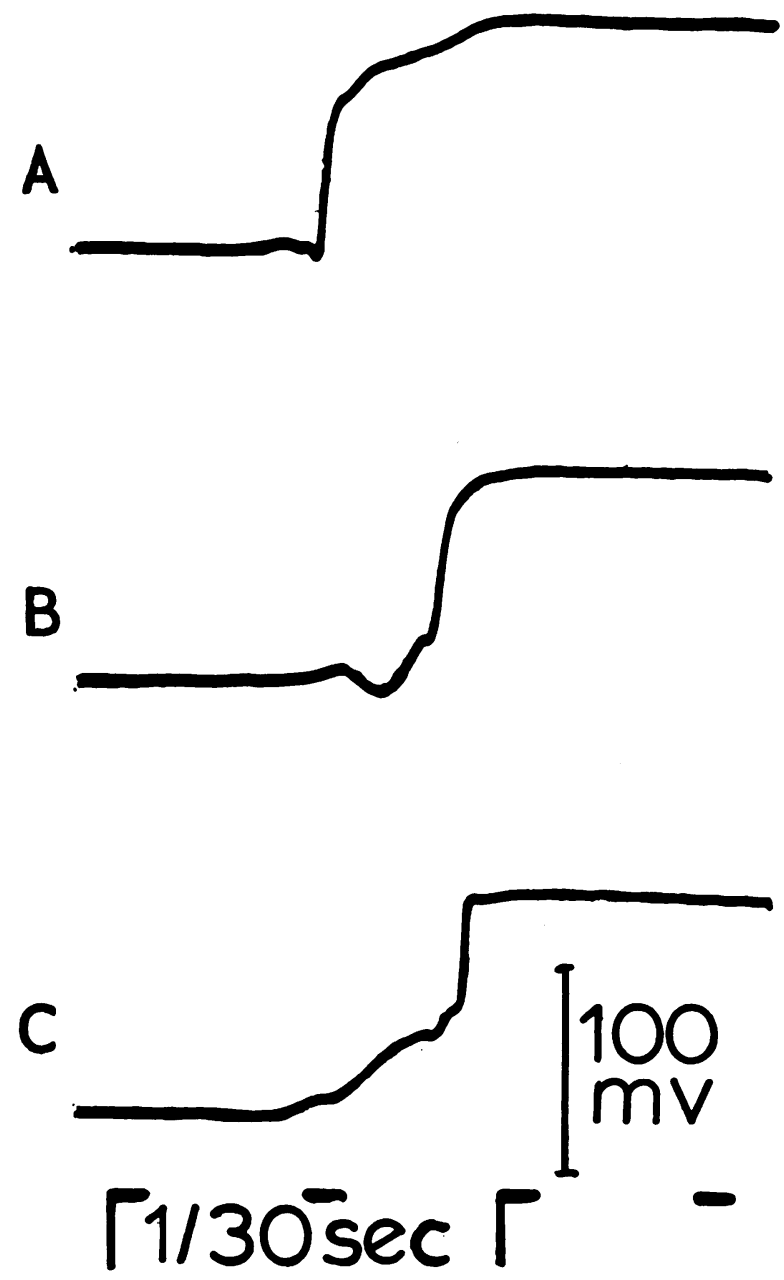

FIG. 1.-Typical monophasic upstrokes obtained from the surface of the guinea-pig ventricle with intracellular electrodes, using a remote reference electrode. The steep rise marks activation of the cell. The irregular gradual rises are ECG contaminants. The steep rise is early in $\mathrm{A}$, intermediate in $\mathrm{B}$ and late in $\mathrm{C}$. 
the approximate duration of the QRS interval of the ECG recorded from the surface of the guineapig ventricle. The duration of the steep part of the monophasic upstroke was approximately $0 \cdot 7$ milliseconds (determined from records with an effective writing speed of 4.5 metres per second). The fact that the total monophasic upstroke had a duration approximately equal to the QRS interval of the surface ECG suggested that the irregularities in it were produced from contamination of the intracellular record with the ECG from the rest of the heart. To obtain a true transmembrane potential, of course, one electrode should be inside the cell and the other immediately outside. For this reason it was decided to try the effect of subtracting the surface ECG recorded from a wire loop $0.5 \mathrm{~mm}$. in diameter which surrounded the tip of the microelectrode from the microelectrode tracing obtained by recording the potential differences between the intracellular electrode and the leg electrode, the leg electrode being used as a reference in both cases (Dower and Geddes, 1960). In effect, this subtraction method meant that potential differences were recorded between the micro-
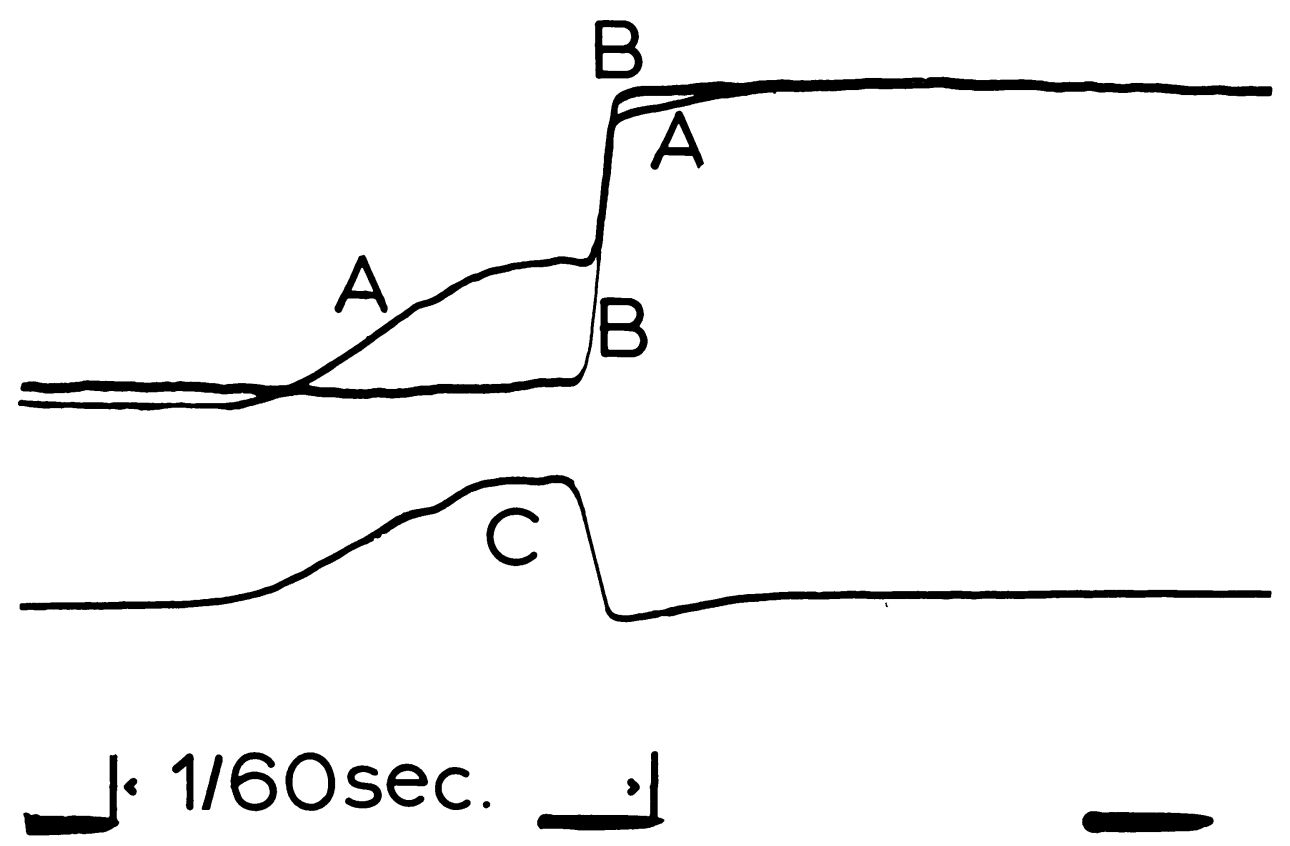

FIG. 2.-Removal of the ECG contaminants from the upstroke of the transmembrane potential curve. The tracing of the potential variations of an intracellular microelectrode with respect to a reference electrode on the leg, $\mathrm{A}$, has subtracted from it the potential variations of a surrounding wire loop electrode with respect to the same reference, $C$, to give a monophasic curve with a sigmoidal upstroke, B-the true transmembrane potential curve.

electrode and the loop electrode on the surface of the heart. The results are shown in Fig. 2: the irregularities of the monophasic upstroke are largely removed and a sigmoidal profile is produced. The total duration of the upstroke is greatly decreased-to between 1.5 and 3 milliseconds (Dower and Geddes, 1960). Without doubt, the sigmoidal upstroke of the monophasic curve now obtained represents activation of the cell penetrated by the microelectrode tip. It is pertinent at this point to ask ourselves where, in fact, the microelectrode tip lies in experiments such as this. We must remember that the microelectrode is held against the surface of the heart by a flexible wire. Although the force exerted by the wire is slight (being merely sufficient to hold the electrode against the heart), when it is concentrated over the extremely minute area of the electrode tip it becomes very large. Now it is not an uncommon experience for microelectrode intracellular recordings to yield monophasic curves which last perhaps 30 seconds or even more. This means that the tip of 
the electrode remains within a cell for this period although the heart is vigorously beating and the microelectrode itself moving with it. This can result only if the microelectrode is held at its sides by being wedged between adjacent cells. It follows, therefore, that the microelectrode itself records not from cells immediately on the surface but from cells beneath the surface. It is common experience to see small dimpling of the surface of the heart at the point of penetration of the electrode. An objection to the use of the ring electrode as an ideal reference for recording transmembrane potentials may now be raised: the ring lies on the surface of the heart but the microelectrode tip lies some distance beneath it. When this discrepancy in distance was not accompanied by a discrepancy in time, i.e. when the cell in which the microelectrode tip lay and the cells on the surface of the heart covered by the ring electrode were activated simultaneously, good sigmoidal upstrokes were obtained. In other cases, i.e. where there was some discrepancy in time, the upstrokes of the monophasic curves showed a step pattern-the tread of the step representing the time interval between activation of the sub-surface and surface regions. That such a time interval could exist between surface and sub-surface regions was indicated by another experiment in which the ring electrode was slid up the shank of the microelectrode so that it no longer pressed on the heart. It was found in one or two experiments in which the microelectrode tip was recording extracellularly (i.e. it had not yet entered a cell) that two separate ECG signals could be obtained from the microelectrode itself: an internal ECG signal from the cell at the tip conducted through the lumen of the microelectrode and an external ECG signal conducted in a fine film of saline passing from cells being activated on the surface of the heart. Subtracting the internal and external ECG signals sometimes left a short spike, the duration of which yielded the time difference between activation of the two regions. Because the loop electrode technique applies only when there is no time difference, we should reject those tracings in which sigmoidal curves show a step for, in these tracings, activation of the cells covered by the ring electrode was different from activation of the cell penetrated by the microelectrode tip.

It is now possible to explain the anomalous results obtained by Sano et al. (1956). We recall in our technique that it is possible to use as grounds for rejection all records which do not yield on subtraction a monophasic curve with a sigmoidal upstroke. If we include such records, we should find, as did Sano et al. (1956), a considerable discrepancy between the steep downward deflection of the surface ECG and the steep upward deflection of the microelectrode monophasic curve. It should be noted here that the paper speeds indicated by the illustrations in the paper by Sano et al. were too slow to indicate the irregularities of the upstroke described here. These authors were presumably unaware that the microelectrode potential curve, normally obtained in vivo using a remote reference, shows considerable ECG contamination.

When transmembrane potentials are obtained from the surface of the guinea-pig ventricle according to the technique described above, we see that we may look upon the upstroke of the monophasic curve as being contained in two separate signals: that obtained from the microelectrode and that obtained from the loop electrode, the same remote reference, such as a leg electrode, being used in each case. This means that the surface ECG obtained from the loop electrode must contain a rapid downward deflection-of an amplitude of perhaps 50 millivolts. Not only must this downward deflection be very rapid but it must be exactly in step with the monophasic curve obtained with the intracellular electrode. Since we accept that the upstroke of the monophasic curves indicates activation, we may take it that the steep downstroke in the surface electrocardiogram likewise indicates activation, and since the slopes are of comparable steepness in many cases, no lack of accuracy would result from using the surface ECG to indicate activation. We have thus shown that there is a component in the surface ECG that can be said to indicate activation of the cells immediately underlying the electrode. We should, therefore, be justified in terming this the intrinsic deflection and, just as we may see differences between the activation of different regions of the ventricular surface indicated by microelectrodes, so also may we see them indicated by the intrinsic deflection in simple surface electrodes.

It has thus been established that Lewis was right in believing that the surface ECG could be used 
to indicate electrical activation, but the literature is somewhat confused as to which part of the surface ECG should properly be considered intrinsic. Lewis used the term intrinsic deflection to indicate the part of the ECG that was generated by the underlying tissue, but there has been some doubt as to which part of the QRS should be considered intrinsic. In an attempt to clarify terminology, the following outline of usage of intrinsic deflection is given. Speaking of the QRS in records obtained with one electrode on the ventricles and the other on the chest wall, Lewis declared, "the prominent spike will be spoken of, therefore, as . . intrinsic deflection" (Lewis et al., 1914). Although he does not specifically say so it is clear that Lewis took his measurements from the apex of this spike because in one of his figures he labels it "in" and the small notch preceding it he labels "ex" for extrinsic deflection, and he has written on the figure "Ex-In $=.0081$ " (Lewis and Rothschild, 1915, Fig. 26). It can be seen from Lewis's figure that the spikes he labelled "In" were each formed by a steep upstroke followed by a more gradual downstroke (Lewis et al., 1914, Fig. 9A, though this applies to the atrium). The polarity he used was such that the steep upstroke indicated a sudden change in the polarity of the electrode on the heart, so that it became more negative with respect to the reference. It must be stressed that by deflection Lewis meant a double movement of the trace, for he identified the intrinsic deflection as a spike. Wilson et al. (1944), from a consideration of the wave-form generated by a migrating dipole passing beneath the exploring electrode, considered "the sudden downward movement that begins with the peak of $R$ " to be the intrinsic deflection and (later) that "the peak of $\mathrm{R}$, therefore, separates the QRS components attributable to muscle activated before, from the QRS components attributable to muscle activated after, the cardiac impulse reached the muscle in contact with the exploring electrode." Wilson differs from Lewis because he speaks of the deflection as a single movement. Furthermore, he reversed the polarity of the records so that the steep upstroke observable in Lewis's records becomes a "sudden downward movement" in those of Wilson. Speaking of the rapid excursion, therefore, we may note that whereas Lewis used its end, Wilson used its beginning to mark immediately underlying activity. However, Wilson being influenced by the work of Cole and Curtis (1938-39) on nerve later changed his position and stated that the end of the rapid downstroke should be used (Wilson et al., 1947). Confusion is likely to arise at this point because Wilson states that Lewis used the beginning of his measurements whereas in fact it was Wilson who used the beginning and Lewis who used the end. Other authors have used their own terms, e.g. Harris referred to "the sharply upbreaking point of the unipolar deflection" (Harris, 1941) but his polarity was the same as Lewis's so he really used the same reference as first suggested by Wilson. Sodi-Pallares et al. (1955) have spoken of "the nadir of the intrinsic deflection" by which they mean the end of the rapid movement, since they use the same polarity as Wilson. It appears conventional today to use the polarity of Wilson and to follow him in applying the term intrinsic deflection to a sudden downward movement of the trace. There is, however, no unanimity as to which part of such a movement should be designated as being the best indication of local activity. Quite recently, Zarday et al. (1959) have applied the term "intrinsic" to both downward and upward deflections in præcordial leads and conclude that such deflections do not indicate local activity.

From the present work we see that the steep negative-going downstroke of the direct ECG coincides with the upstroke of the transmembrane potential curves of the underlying cell or cells. This must be, therefore, the intrinsic deflection. This agrees with the view of Durrer et al. (1954). It is not necessary to indicate whether the intrinsic deflection forms the downstroke of an $R$ or the upstroke of an S wave for it may constitute either or both (Dower and Geddes, 1960). We may now give an explanation for the remarkable variation in steepness of the intrinsic deflection noted by Lewis in which over the right ventricle, intrinsic deflections are considerably less steep than those over the left (Lewis and Rothschild, 1915). On the left ventricle, the intrinsic deflection and the deflection produced by the electric field generated by other parts of the heart supplement each other, being in the same direction, and the intrinsic deflections appear steep. On the right ventricle, it often happens that the downward intrinsic deflection occurs during the period when the deflection produced by the rest of the heart is upward so that the observed intrinsic deflection is much less steep. 
If we admit, as we must, that an electrode placed on the surface of the heart records electrical events taking place immediately beneath it, in addition to the electrical activity of the heart as a whole, we cannot deny that such an electrode might record an abnormal occurrence which would not appear, or would be lost in, the signals obtained from remote points. The implications of this conclusion for præcordial electrocardiographic leads must await the unequivocal demonstration of the existence of intrinsicoid deflections in these leads. Until then, it would seem incautious to neglect the possibility that præcordial leads, at least to some extent, record events occurring immediately subjacent to them.

The restoration of the intrinsic deflection is of importance only in so far as it may influence concepts in electrocardiography and vectorcardiography. As methods of indicating ventricular activation, the use of the intrinsic deflection or the upstroke of the true transmembrane potential curve appear to offer no theoretical advantage over differential electrodes which are technically much easier to use (Scher et al., 1953; Durrer and van der Tweel, 1953).

\section{Summary}

The intrinsic deflection of Lewis has been variously defined and has been rejected by some as an indication of activity beneath an electrode on the surface of the heart. The use of intracellular electrodes, a surrounding wire loop electrode and fast recording speeds has, however, shown that the surface electrocardiogram does, in fact, present a negative deflection that is produced by immediately subjacent cells and which may, therefore, correctly be termed "intrinsic". It is more marked on the left than on the right due to the electric field generated by the heart as a whole. The importance of this finding relates to the possibility of the existence of appreciable intrinsicoid deflections in præcordial leads.

\section{REFERENCES}

Cole, K. S., and Curtis, H. J. (1938-39). J. gen. Physiol., 22, 649.

Cranefield, P. F., and Hoffman, B. F. (1958). Physiol. Rev., 38, 41.

Dower, G. E., and Geddes, M. A. (1960). Amer. J. Physiol., 198, 975.

- and Osborne, J. A. (1958). Amer. J. Physiol., 195, 396.

Duchosal, P. W., and Sulzer, R. (1949). La Vectorcardiographie. Basel, S. Karger.

Durrer, D., and van der Tweel, L. H. (1953). Amer. Heart J., 46, 683.

- - (1954). Amer. Heart J., 47, 192.

Frank, E. (1959). In Electrophysiology of the Heart. New York Academy of Science, p. 980.

Harris, A. S. (1941). Amer. J. Physiol., 134, 319.

Lewis, T., Meakins, J., and White, P. D. (1914). Phil. Trans. B., 205, 375.

-, and Rothschild, M. A. (1915). Phil. Trans. B, 206, 181.

McFee, R., and Parungao, A. (1959). Amer. Heart J., 58, 582.

MacLeod, A. G., Wilson, F. N., and Barker, P. S. (1930). Proc. Soc. exp. Biol., $27,586$.

Pipberger, H. V., Bialek, S. M., Perloff, J. K., and Schnaper, H. W. (1961). Amer. Heart J., 61, 34.

Sano, T., Ono, M., and Shimamoto, T. (1956). Circulation Res., 4, 444.

-, Takayama, N., and Shimamoto, T. (1959). Amer. Heart J., 57, 606.

Scher, A. M., Young, A. C., Malingren, A. L., and Paton, R. P. (1953). Circulation Res., 1, 539.

Schmitt, O. H., and Simonson, E. (1955). Arch. intern. Med., 96, 574.

Sodi-Pallares, D., Bisteni, A., Medrano, G. A., and Cisneros, F. (1955). Amer. Heart J., 49, 587.

Wilson, F. N., Johnston, F. D., Rosenbaum, F. F., Erlanger, H., Kossmann, C. E., Hecht, H., Cotrim, N., De Oliveira, R. M., Scarsi, R., and Barker, P. S. (1944). Amer. Heart J., 27, 19.

-_, Rosenbaum, F. F., and Johnston, F. D. (1947). Advances in Internal Medicine, vol. 2, New York, Interscience, p. 13.

Woodbury, J. W., and Brady, A. J. (1956). Science, 123, 100.

Zarday, I., Kristoff, G., and Solti, F. (1959). Acta Cardiol., 14, 158. 